\title{
MicroRNA-130a is highly expressed in the esophageal mucosa of achalasia patients
}

\author{
HIROYUKI SHOJI $^{1}$, HAJIME ISOMOTO ${ }^{1,2}$, AKIRA YOSHIDA ${ }^{1,3}$, HARUO IKEDA $^{3,4}$, \\ HITOMI MINAMI ${ }^{1}$, TSUTOMU KANDA ${ }^{1}$, SHIGETOSHI URABE ${ }^{1}$, KAYOKO MATSUSHIMA $^{1}$, \\ FUMINAO TAKESHIMA $^{1}$, KAZUHIKO NAKAO ${ }^{1}$ and HARUHIRO INOUE ${ }^{3,4}$ \\ ${ }^{1}$ Department of Gastroenterology and Hepatology, Graduate School of Biomedical Sciences, \\ Nagasaki University, Nagasaki 852-8501; ${ }^{2}$ Division of Medicine and Clinical Science, \\ Department of Multidisciplinary Internal Medicine, Tottori University School of Medicine, Yonago, Tottori 683-8504; \\ ${ }^{3}$ Digestive Disease Center, Showa University Northern Yokohama Hospital, Yokohama, Kanagawa 224-8503; \\ ${ }^{4}$ Digestive Disease Center, Showa University Koto Toyosu Hospital, Koto-ku, Tokyo 135-8577, Japan
}

Received December 8, 2015; Accepted January 13, 2017

DOI: $10.3892 /$ etm.2017.4598

\begin{abstract}
Esophageal achalasia is considered as a risk factor of esophageal cancer. The etiologies of esophageal achalasia remain unknown. Peroral endoscopic myotomy (POEM) has recently been established as a minimally invasive method with high curability. The aims of the present study were to identify the microRNAs (miRs) specific to esophageal achalasia, to determine their potential target genes and to assess their alteration following POEM. RNA was extracted from biopsy samples from middle esophageal mucosa and analyzed using a microarray. Differentially expressed miRs in achalasia patients compared with control samples were identified and analyzed using reverse transcription-quantitative polymerase chain reaction (RT-qPCR). Correlations between specific miR expression levels and the patients' clinical background were also investigated. In addition, alterations of selected miR expression levels before and after POEM were analyzed. The results of RT-qPCR analysis demonstrated that the miR-130a expression levels were significantly higher in patients with achalasia $(\mathrm{P}<0.0001)$. In addition, miR-130a expression was significantly correlated with male sex and smoking history in patients with achalasia. However, no significant change in
\end{abstract}

Correspondence to: Dr Hiroyuki Shoji, Department of Gastroenterology and Hepatology, Graduate School of Biomedical Sciences, Nagasaki University, 1-7-1 Sakamoto, Nagasaki 852-8501, Japan

E-mail: jr11dh14mm15@gmail.com

Dr Hajime Isomoto, Division of Medicine and Clinical Science, Department of Multidisciplinary Internal Medicine, Tottori University School of Medicine, 36-1 Nishi-cho, Yonago, Tottori 683-8504, Japan E-mail: isomoto@med.tottori-u.ac.jp

Key words: esophageal achalasia, etiology, microRNA, peroral endoscopic myotomy, esophageal cancer
miR-130a expression was observed between before and after POEM. In conclusion, miR-130a is highly expressed in the esophageal mucosa of patients with achalasia and may be a biomarker of esophageal achalasia.

\section{Introduction}

Esophageal achalasia is motility disorder resulting from incomplete relaxation of the lower esophageal sphincter (LES) and the loss of normal peristalsis (1-3). The predominant symptoms of esophageal achalasia are dysphagia and regurgitation. Patients with achalasia are diagnosed by self-reported symptoms, endoscopy and esophagography. Achalasia is a rare and chronic disease that can occur at any age (2), and is considered as a risk factor of esophageal cancer $(1,3)$. The risk of developing squamous cell carcinoma in achalasia patients was demonstrated to be increased by 33 -fold compared with that in the general population (4). However, the etiology of esophageal achalasia, particularly the process leading to carcinogenesis, remains largely unknown.

Persistent esophageal distension with retention of food and fluids, bacterial overgrowth, and impaired clearance of regurgitated acid and gastric contents are known to lead to chronic inflammation and to passively cause dysplasia and carcinoma (5-7). Therapy for esophageal achalasia aims to reduce food stagnation (8). Various treatments have been devised for achalasia patients, including medication, balloon dilatation and surgery. Endoscopic balloon dilatation remains a widely performed treatment due to the relative noninvasiveness and simplicity of the procedure; however, it has a relatively low success rate and often requires multiple treatment sessions (9). Peroral endoscopic myotomy (POEM) has been recently established as a minimally invasive procedure with high success rate $(10,11)$. Inoue et al $(10)$ reported that, in short-term results, there was no recurrence subsequent to the POEM procedure in 17 cases of achalasia. POEM can be successfully and safely performed by skilled endoscopists, and effectively ameliorates dysphagia symptoms. Manometric pressure studies have also 
demonstrated significant improvement in the lower esophageal sphincter pressure following POEM (11). In addition, POEM substantially decreased the Ki-67-positive and P53-positive ratios in esophageal epithelia. Thus, POEM appears to reduce the risk of esophageal carcinogenesis (11).

MicroRNAs (miRs) are small non-coding RNAs that negatively regulate gene expression via translational repression or messenger RNA degradation (12). Over 2,800 miRs have been identified in humans, with each individual miR predicted to target multiple genes based on the seed sequence matching their 3'-untranslated regions (UTRs) (13). miRs are involved in biological and pathological processes, including cell differentiation, proliferation, apoptosis and metabolism (14), and they are emerging as highly tissue-specific biomarkers with potential clinical applicability for defining cancer type and origin $(15,16)$. Accumulating evidence has indicated that deregulation of miRs is associated with human malignancies, and suggested that miRs may have a causal role in tumor initiation and progression, since they can function as oncogenes or tumor suppressors (17). Indeed, previous studies have indicated distinct differences in $\mathrm{miR}$ expression patterns between squamous cell carcinoma and adenocarcinoma in esophageal and other cancer types (18-20). For instance, Kimura et al (21) reported that the highest expression of miR-205 was identified in both benign and malignant squamous epithelia, including in esophageal squamous cell carcinoma, whereas a lower expression was observed in cell lines and tissues other than squamous epithelia. Additionally, miR-21, which is an oncogenic miR in various malignancies, was also upregulated in esophageal squamous cell carcinoma compared with its expression in paired normal squamous epithelia (21). There is also growing evidence regarding the pathogenic roles of miRs in immune and inflammatory disorders, including esophagitis. For example, elevated miR-143, miR-145 and miR-205 expression levels were observed in the esophageal squamous mucosa of individuals with ulcerative esophagitis, where they may be involved in regulating epithelial restoration in response to injury caused by gastro-esophageal reflux (22).

As mentioned earlier, it is generally accepted that achalasia is a pre-malignant disorder that is possibly caused by longstanding mucosal inflammation due to persistent stasis of food (23). Nevertheless, there is little information regarding the miR expression profile in achalasia. Therefore, the aims of the present study were to identify the miR expression specific to the esophageal mucosa of achalasia patients, to determine potential target genes of these miRs and to assess the alteration of miRs following POEM.

\section{Materials and methods}

Patients and clinical samples. A total of 29 achalasia patients who visited the Showa University Northern Yokohama Hospital (Yokohama, Japan) between October 2011 and June 2012 were enrolled into the current study. Patients with any severe underlying illness, such as cancer, or those who could not tolerate general anesthesia were excluded. A total of 14 males and 15 females aged between 23 and 85 years $($ mean=46) were enrolled. There were 15 smokers and 13 non-smokers (1 unknown). They were all known to have achalasia. A total of 23 patients suffered from straight-type achalasia (the meandering of the longitudinal axis of the esophagus appears weak on barium esophagogram) and 6 patients suffered with sigmoid-type achalasia (the meandering of the longitudinal axis of the esophagus appears strong on barium esophagogram). The degree of esophageal dilatation was grade I in 7 patients and grade II in 22 patients, as defined by the Descriptive Rules for Achalasia of the Esophagus (24). A total of 4 healthy subjects with no severe underlying illnesses were enrolled in the present study (2 male, 2 female; aged 63-68 years). Subsequent to obtaining informed consent, 2 biopsy samples were collected from the middle esophageal mucosa of each patient during esophagoscopy before POEM, and were immediately placed into $1 \mathrm{ml}$ RNAlater reagent (Ambion; Thermo Fisher Scientific, Inc., Waltham, MA, USA) and stored at $-80^{\circ} \mathrm{C}$ for subsequent RNA isolation. All examinations were conducted according to the 6th Good Clinical Practice guidelines and the Declaration of Helsinki, and were approved by the Nagasaki and Showa University Ethics Committees.

POEM. All patients underwent POEM, which was performed as follows and the procedures were performed under general anesthesia with positive pressure ventilation. A submucosal tunnel was formed from the central esophagus to the esophago-gastric junction beyond, using a technique similar to endoscopic mucosal dissection (ESD) (25). An incision was subsequently made of the circular muscle bundle from the entrance to the LES. The incision mucosal invasion was closed with a hemostatic clip (8).

RNA extraction. Total RNA, including small RNA, was extracted from the tissue samples using the mirVana miRNA isolation kit (AM1560; Ambion; Thermo Fisher Scientific, Inc.), and the total RNA was quantified using a Nanodrop-1000 spectrophotometer (Nanodrop Technologies, Wilmington, DE, USA). Next, the total RNA was purified using the miRNeasy mini kit (cat. no. 217004; Qiagen, Hilden, Germany), and the quality of the total RNA was determined by UV absorption measurement and on Agilent 2100 Bioanalyzer (Agilent Technologies, Santa Clara, CA, USA).

miR array hybridization and analysis. In order to identify the $\operatorname{miR}(\mathrm{s})$ specific to the esophageal mucosa of achalasia, total RNA was extracted from the biopsy mucosal tissues of 8 representative cases of achalasia and from those of 4 healthy volunteer controls. Following DNase treatment, the isolated RNA samples were subjected to comprehensive analysis of miR expression patterns using microarray-based technology. These analyses were performed by Hokkaido System Science Co., Ltd. (Sapporo, Japan) using the SurePrint G3 Human 8x60 K microarray version 2.0 (Agilent Technologies) and $50 \mathrm{ng}$ aliquots of each total RNA sample. The scan was performed using the Agilent Technologies Microarray Scanner (Agilent Technologies) at $3 \mu \mathrm{m}$ resolution, and each spot was digitized using Agilent Feature Extraction version 10.7.3.1 software. To identify the miRs that were differentially expressed in esophageal mucosa, data were imported to GeneSpring GX version 10.7.3.1 (Agilent Technologies) and analyzed; a feature is considered detected if the signal is three-fold greater than the error. The differences in miR expression were considered as statistically significant if the 


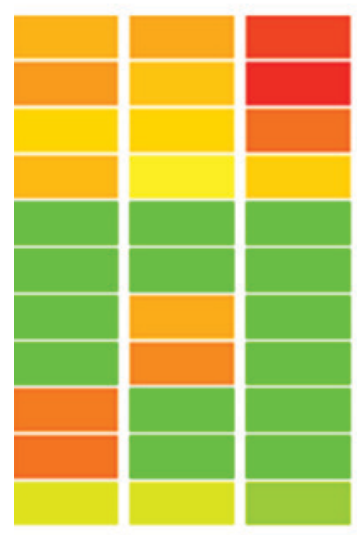

Healthy control group

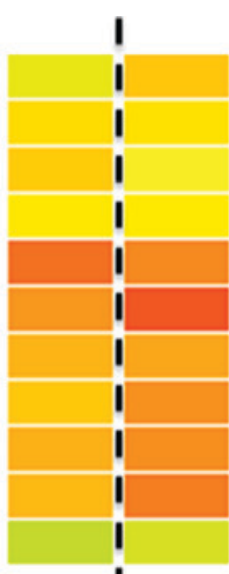

I
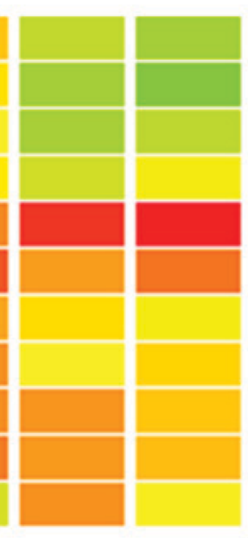

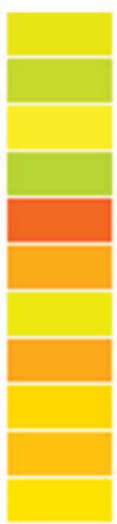

Achalasia group
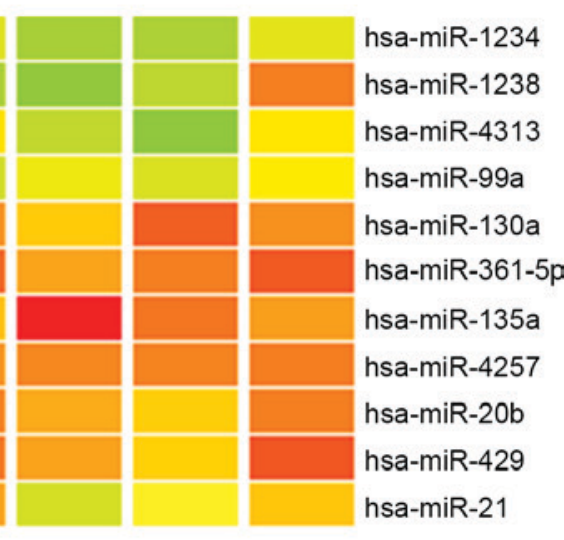

Figure 1. Microarray analysis of miRNA expression in esophageal achalasia patients. The microarray results indicated high expression of miR-361-5p and miR-130a in the esophageal mucosa of esophageal achalasia patients compared with healthy controls. The red and orange colors indicate high expression, bright green indicates low expression.

fold change in expression values was $>2.0$ and $\mathrm{P}<0.05$ using a Student's t-test.

Reverse transcription-quantitative polymerase chain reaction $(R T-q P C R)$ analysis. The expression levels of miRs that showed significant differences based on the microarray results were analyzed using RT-qPCR. Briefly, cDNA was prepared from total RNA using the High Capacity cDNA Reverse Transcription kit (cat. no. 4374966; Applied Biosystems; Thermo Fisher Scientific, Inc.) and the indicated TaqMan small RNA assay kit (Applied Biosystems; Thermo Fisher Scientific, Inc.). The RT reactions were performed in a solution containing $10 \mathrm{ng}$ total RNA, 1X RT primer, 1X RT buffer, $1 \mathrm{mM}$ dNTP, 50 units MultiScribe Reverse Transcriptase and 3.8 units of RNase inhibitor. Nuclease-free water was added to bring the solution to a total volume of $15 \mu l$. The reactions were run on the TGradient thermocycler (Biometra $\mathrm{GmbH}$, Göttingen, Germany) at $16^{\circ} \mathrm{C}$ for $30 \mathrm{~min}$, followed by $42^{\circ} \mathrm{C}$ for $30 \mathrm{~min}$, and then $85^{\circ} \mathrm{C}$ for $5 \mathrm{~min}$. Subsequently, qPCR reactions were performed in a solution containing $1.33 \mu \mathrm{l}$ RT products with 1X TaqMan Universal Master Mix II without Uracil-N glycosylase (UNG) (cat. no. 4440040; Applied Biosystems; Thermo Fisher Scientific, Inc.) and $1 \mu \mathrm{l}$ of each of the TaqMan small RNA assay primers. Each Taqman small RNA primer set contained primers for the analysis of has-miR-361-5p, has-miR-130a and RNU6B (cat. nos. 000554, 000454 and 001093, respectively; Applied Biosystems; Thermo Fisher Scientific, Inc.) Nuclease-free water was added to obtain a solution with total volume of $20 \mu \mathrm{l}$. All reactions were run in triplicate using the LightCycler 480 II (Roche Diagnostics, Basel, Switzerland). The thermal cycling reactions were initiated at $95^{\circ} \mathrm{C}$ for $10 \mathrm{~min}$, followed by 45 cycles of $95^{\circ} \mathrm{C}$ for $15 \mathrm{sec}$, and $60^{\circ} \mathrm{C}$ for $1 \mathrm{~min}$. The cycle passing threshold $(\mathrm{Cq})$ was recorded for each candidate $\mathrm{miR}$, and the $2^{-\Delta \Delta \mathrm{Cq}}$ method was used with RNU6B as the endogenous control for data normalization (12).

In order to determine potential target genes of miR-130a, RT-qPCR was performed to determine changes in the mRNA expression in the mucosa of achalasia patients compared with that in healthy controls. cDNA was prepared from total
RNA using the High Capacity cDNA Reverse Transcription kit (cat. no. 4374966; Applied Biosystems; Thermo Fisher Scientific, Inc.). The RT reactions were performed in a solution containing $500 \mathrm{ng}$ total RNA, 1X Random Primer, 1X RT Buffer, 4 mM dNTP, 50 units MultiScribe Reverse Transcriptase and 20 units RNase Inhibitor. Nuclease-free water was added to obtain a total volume of $20 \mu \mathrm{l}$. The reactions were run on the TGradient thermocycler (Biometra) at $25^{\circ} \mathrm{C}$ for $10 \mathrm{~min}$, followed by $37^{\circ} \mathrm{C}$ for $120 \mathrm{~min}$, and $85^{\circ} \mathrm{C}$ for $5 \mathrm{~min}$. Next, qPCR reactions were performed in a solution containing $4 \mu \mathrm{l}$ RT products with $1 \mathrm{X}$ TaqMan Universal Master Mix II without UNG (cat. no. 4440040; Applied Biosystems; Thermo Fisher Scientific, Inc.) and $1 \mu \mathrm{l}$ of each of the TaqMan mRNA assay primer sets. Nuclease-free water was added to bring the total volume up to $20 \mu \mathrm{l}$. Of the significantly altered genes, in silico Target Scan (version 6.2; Whitehead Institute for Biomedical Research, Cambridge, MA, USA) prediction indicated that myotubularin related protein 10 (MTMR10) and WNK lysine deficient protein kinase 1 (WNK1) may be candidate target genes of miR-130a, based on the seed sequence matches in their 3'-UTRs. The TaqMan mRNA primer sets used were for the amplification of the mRNAs for MTMR10, WNK1 and glyceraldehyde 3-phosphate dehydrogenase (GAPDH) (cat. nos.Hs01107504_m1,Hs00219183_m1 and Hs99999905_m1, respectively; Applied Biosystems; Thermo Fisher Scientific, Inc.). Reactions were run on the LightCycler 480 II (Roche Diagnostics), and thermal cycling was initiated at $95^{\circ} \mathrm{C}$ for $10 \mathrm{~min}$, followed by 45 cycles of $95^{\circ} \mathrm{C}$ for $15 \mathrm{sec}$ and $60^{\circ} \mathrm{C}$ for $1 \mathrm{~min}$. $\mathrm{Cq}$ values were recorded for each candidate mRNA, and the $2^{-\triangle \Delta \mathrm{Cq}}$ method was performed, using GAPDH as the endogenous control for data normalization.

Statistical analysis. The differences between groups were analyzed using the unpaired, one-tailed, Student's t-test. Data are expressed as the mean \pm standard error. Differences were considered to be statistically significant at $\mathrm{P}<0.05$. Multiple regression analyses were also performed. All data were analysed using StatFlex version 6 (Artech Information Systems LLC, Morristown, NJ, USA). 

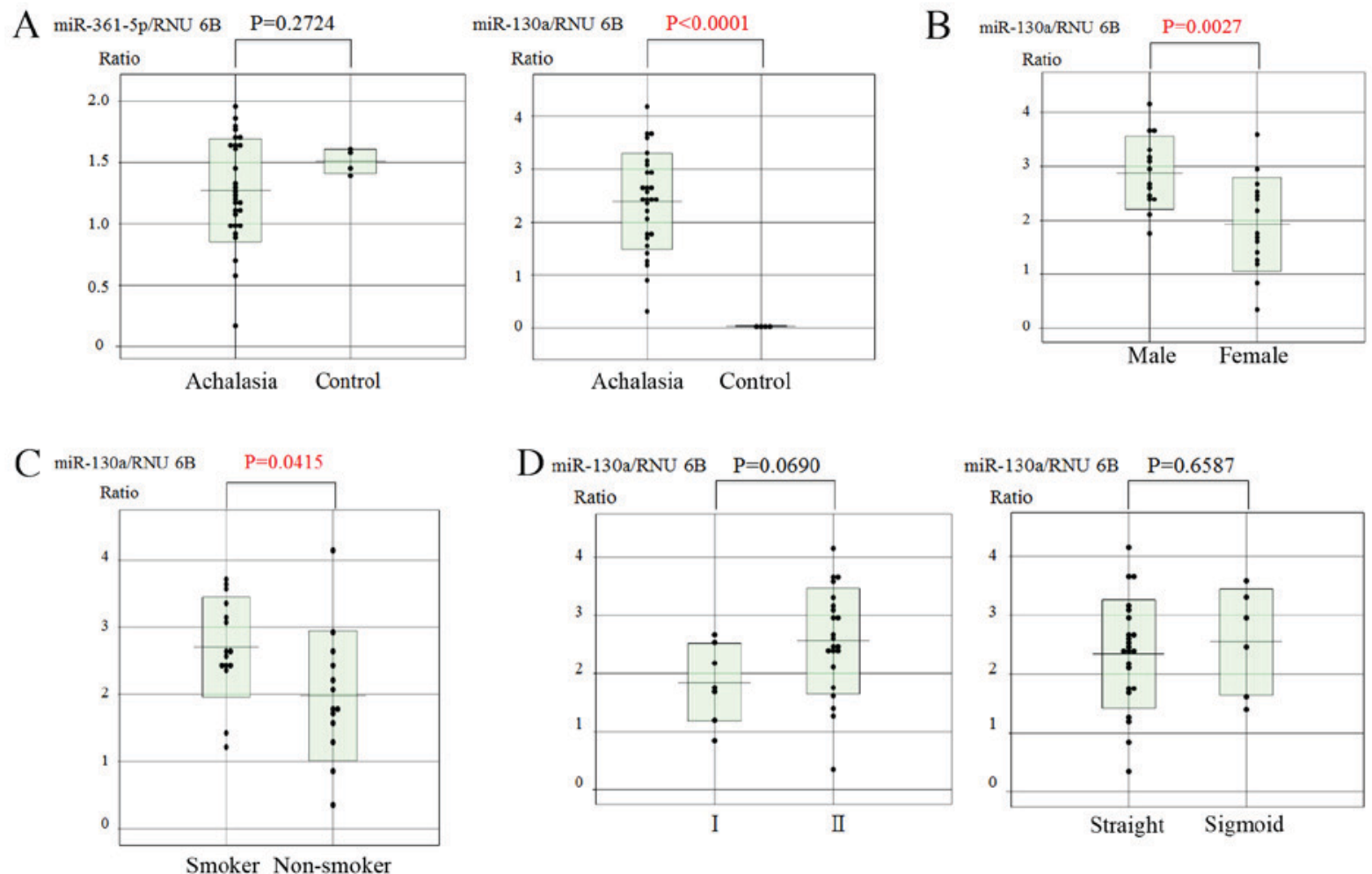

Figure 2. Expression levels of miR-361-5p and miR-130a in achalasia patients, and the association of miR-130-a with sex, smoking and esophageal dilation. (A) Quantitative analysis of miR-361-5p and miR-130a expression in achalasia patients and healthy subjects, analyzed using reverse transcription-quantitative polymerase chain reaction. The data are shown relative to RNU6B expression. miR-130a demonstrated a significantly higher expression in achalasia patients vs. control healthy subjects $(\mathrm{P}<0.0001)$. (B) Significant correlation was observed between the expression of miR-130a and male sex (vs. female sex; $\mathrm{P}=0.0027)$ in achalasia patients. (C) Significant correlation was detected between the expression of miR-130a and smoking habit (vs. non-smoker; $\mathrm{P}=0.0415)$ in achalasia patients. (D) No significant correlation was detected between the expression of miR-130a and the type of achalasia or the degree of esophageal dilatation. miR, microRNA.

\section{Results}

Patient characteristics. Of the patients enrolled in the present study, 23 suffered from straight-type achalasia and 6 with sigmoid-type achalasia. The degree of esophageal dilatation was grade I in 7 patients and grade II in 22 patients (24). The mean disease duration was 60 months, ranging between 5 and 564 months.

Microarray analysis results. The microarray analysis of miR expression levels in the esophageal mucosa of achalasia indicated that miR-361-5p and miR-130a were significantly (>2-fold) overexpressed in the esophageal mucosa of achalasia patients when compared with the controls (Fig. 1). Subsequently, RT-qPCR was used to quantify the expression levels of these two miRs in biopsy specimens of achalasia patients and controls. The results revealed that only the expression of miR-130a was significantly higher in achalasia patients compared with that in healthy subjects $(\mathrm{P}<0.0001$; Fig. 2A).

Correlation of miR-130a expression with various parameters. The correlation between the miR-130a expression and the background characteristics of the patients was then analyzed. Significant correlations were observed between the expression levels of miR-130a and sex, with males having significantly higher levels than females $(\mathrm{P}=0.0027$; Fig. 2B)

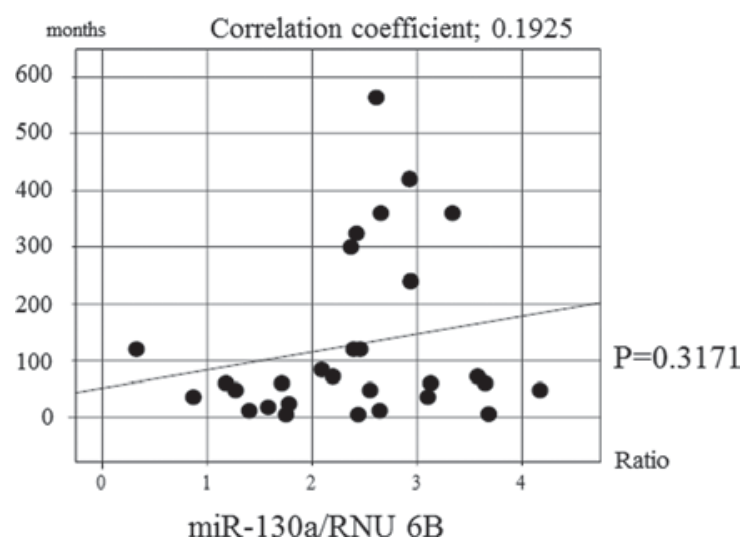

Figure 3. Correlation analysis between miR-130a expression and the disease duration in achalasia patients. The results indicated no significant correlation. miR, microRNA.

and smoker status (elevated in smokers vs. non-smoker; $\mathrm{P}=0.0415$; Fig. 2C) in achalasia patients. However, there were no correlations between miR-130a expression and the degree of esophageal dilatation or the type of achalasia (Fig. 2D). In addition, there was no significant correlation between the expression of miR-130a and the disease duration in achalasia patients (Fig. 3). Multiple regression analysis demonstrated that there was a significant correlation between miR-130a expression and smoking $(\mathrm{P}=0.0084$; data not shown). 


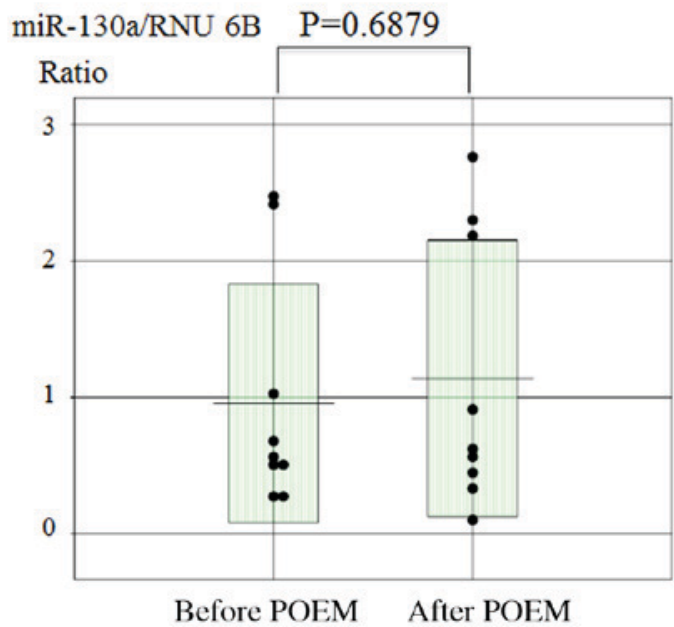

\section{miR-130a/RNU 6B}

Ratio

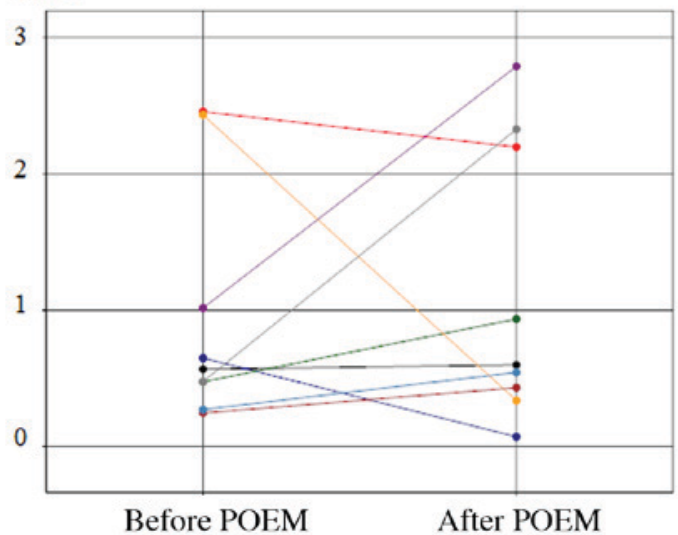

Figure 4. miR-130a expression prior to and following the POEM procedure in achalasia patients analyzed using reverse transcription-quantitative polymerase chain reaction. No significant change was observed in miR-130a expression before and after POEM. miR, microRNA; POEM, peroral endoscopic myotomy.
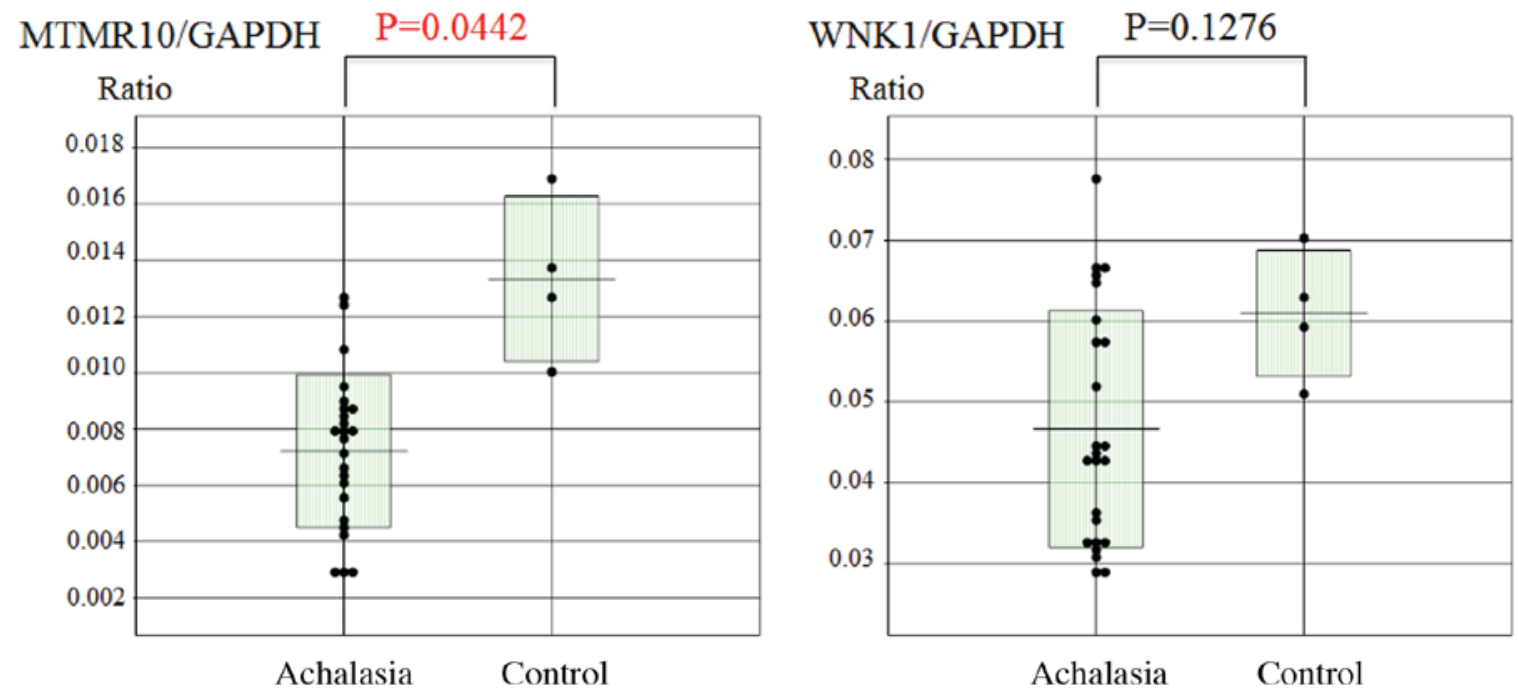

Figure 5. Analysis of the association of mRNA expression levels of MTMR10 and WNK1 with achalasia. A significant correlation with MTMR10 levels was observed in the achalasia patients, as compared with the control group $(\mathrm{P}=0.0442)$. MTMR10, myotubularin related protein 10; WNK1, WNK lysine deficient protein kinase 1 .

Furthermore, no significant change in miR-130a expression was observed prior to and following the POEM procedure in achalasia patients (Fig. 4).

cDNA array analysis results. In order to determine potential target genes of miR-130a, a cDNA array analysis was performed to determine changes in gene expression in the mucosa tissue of achalasia patients compared with the healthy controls. This analysis indicated that there were 845 genes that were upregulated 1.5-fold and 969 genes that were downregulated by 1.5 -fold in achalasia mucosa compared with the control. The levels of these genes were substantially decreased in achalasia patients according to the results of comprehensive cDNA microarray.

Subsequently, the esophageal mucosal mRNA expression levels of MTMR10 and WNK1 were analyzed using RT-qPCR. The mucosal MTMTR10 mRNA levels, but not WNK1, were

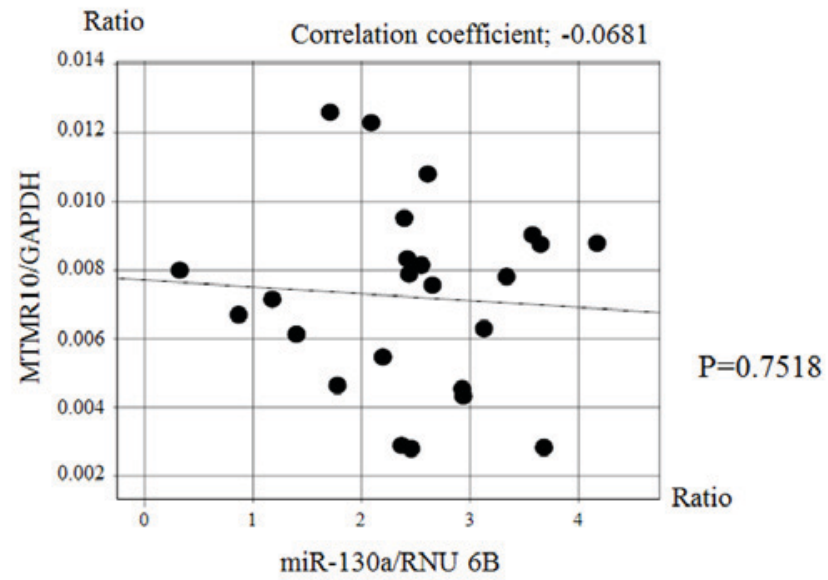

Figure 6. Correlation analysis between the expression of miR-130a and the mRNA expression of MTMR10 indicated no significant correlation. miR, microRNA; MTMR10, myotubularin related protein 10. 
significantly decreased in achalasia patients compared with those in the controls ( $\mathrm{P}=0.0442$; Fig. 5). However, there was no significant correlation between the expression of miR-130a and that of MTMR10 (Fig. 6).

\section{Discussion}

The present study demonstrated that miR-130a was highly expressed in the esophageal mucosa of achalasia patients and that smoking history was associated with a high expression of miR-130a. The miR-130a gene is located at the chromosomal position 11q12, which is close to the region 11q13 that is frequently amplified in cancer (26-29). In fact, miR-130a serves an important role in multiple types of tumors. For instance, miR-130a has been reported to be overexpressed in non-small-cell lung carcinoma (30). Increased expression of miR-130a is strongly associated with lymph node metastasis and poor prognosis of this carcinoma (30). By contrast, miR-130a is downregulated in prostate carcinomas and jointly suppresses two major oncogenic pathways with miR-203 and miR-205 (31). miR-130a also increases drug resistance by regulating RUNX3 and Wnt signaling in cisplatin-treated hepatocellular carcinoma cells (32), while upregulation of miR-130a has been associated with MDR1/P- glycoprotein-mediated drug resistance in ovarian cancer cells (33). Acunzo et al (34) reported that miR-130a was able to target Met and induce TNF-related apoptosis-inducing ligand sensitivity in non-small-cell lung carcinomas by downregulating miR-221 and miR-222 .

In the current study, no significant change in miR-130a expression was observed between before and after POEM. Although POEM may be one option to reduce patient suffering and decrease the risk of future carcinogenesis (8), it is unable to completely prevent achalasia patients from developing esophageal cancer. Several studies have reported the association of smoking with miRs, as well as with lung diseases (35) and various types of cancer (36), including esophageal cancer (12). In the present study, the results suggested that smoking history may be associated with the expression level of miR-130a. However, we were unable to compare the expression of miR-130a in healthy non-smokers, healthy smokers, achalasia non-smokers and achalasia smokers in the current study, and therefore further studies are warranted.

The myotubularin gene (MTM1) was identified as a gene mutated in X-linked myotubular myopathy (37). A subgroup of genes in the myotubularin family encodes proteins that contain substitutions of residues within the $\mathrm{C}(\mathrm{X})_{5} \mathrm{R}$ active site motif and are catalytically inactive. Of the 14 known MTM related (MTMR) human genes, 6 (MTMR5, MTMR9, MTMR10, MTMR11, MTMR12 and MTMR13) encode inactive proteins, whereas the function of MTMR10 is largely unknown (38). In the current study, miR-130a and MTMR10 expression were not correlated at the mRNA level; however, it is possible that they are associated at the protein level. However, the association between eshophageal achalasia and MTMR10 remains unclear, thus further studies are also warranted in this regard.

In conclusion, miR-130a is highly expressed in the esophageal mucosa of esophageal achalasia. Furthermore, smoking history may be associated with the expression level of miR-130a. Therefore, miR-130a may be a useful mucosal biomarker of esophageal achalasia.

\section{References}

1. Leeuwenburgh I, Scholten P, Alderliesten J, Tilanus HW, Looman CW, Steijerberg EW and Kuipers EJ: Long-term esophageal cancer risk in patients with primary achalasia: A prospective study. Am J Gastroenterol 105: 2144-2149, 2010.

2. Gockel I, Muller M and Schumacher J: Achalasia-a disease of unknown cause that is often diagnosed too late. Dtsch Arztebl Int 109: 209-214, 2012.

3. Zendehdel K, Nyrén O, Edberg A and Ye W: Risk of esophageal adenocarcinoma in achalasia patients, a retrospective cohort study in Sweden. Am J Gastroenterol 106: 57-61, 2011.

4. Meijssen MA, Tilanus HW, van Blankenstein M, Hop WC and Ong GL: Achalasia complicated by esophageal squamous cell carcinoma: A prospective study in 195 patients. Gut 33: 155-158, 1992.

5. Porschen R, Molsberger G, Kühn A, Sarbia M and Borchard F: Achalasia-associated squamous cell carcinoma of the esophagus: Flow-cytometric and histological evaluation. Gastroenterology 108: 545549, 1995.

6. Streitz JM Jr, Ellis FH Jr, Gibb SP and Heatley GM: Achalasia and squamous cell carcinoma of the esophagus: Analysis of 241 patients. Ann Thorac Surg 59: 1604-1609, 1995.

7. West RL, Hirsch DP, Bartelsman JF, de Borst J, Ferwerda G, Tytqat GN and Boeckxstaens GE: Long term results of pneumatic dilation in achalasia followed for more than 5 years. Am J Gastroenterol 97: 1346-1351, 2002.

8. Minami H, Yamaguchi N, Matsushima K, Akazawa Y, Ohnita K, Takeshima F, Nakayama T, Hayashi T, Inoue H, Nakao K and Isomoto $\mathrm{H}$ : Improvement of endocytoscopic findings after per oral endoscopic myotomy POEM) in esophageal achalasia; does POEM reduce the risk of developing esophageal carcinoma? Per oral endoscopic myotomy, endocytoscopy and carcinogenesis. BMC Gastroenterol 13: 22, 2013.

9. Campos GM, Vittinqhoff E, Rabl C, Takata M, Gadenstätter M, Lin F and Ciovica R: Endoscopic and surgical treatments for achalasia: A systematic review and meta-analysis. Ann Surg 249: 45-57, 2009.

10. Inoue H, Minami H, Kobayashi Y, Sato Y, Kaga M, Suzuki M, Satodate H, Okada N, Itoh H and Kudo S: Peroral endoscopic myotomy (POEM) for esophageal achalasia. Endoscopy 42: 265-271, 2010.

11. Minami H, Isomoto $\mathrm{H}$, Yamaguchi N, Matsushima K, Akazawa $Y$, Ohnita K, Takeshima F, Inoue H and Nakao K: Peroral endoscopic myotomy for esophageal achalasia: Clinical impact of 28 cases. Dig Endosc 26: 43-51, 2014.

12. Matsushima $K$, Isomoto $H$, Yamaguchi $N$, Inoue $N$, Machida $H$, Nakayama T, Hayashi T, Kunizaki M, Hidaka S, Nagayasu T, et al: MiRNA-205 modulates cellular invasion and migration via regulating zinc finger E-box binding homeobox 2 expression in esophageal squamous cell carcinoma cells. J Transl Med 9: 30, 2011.

13. Carthew RW and Sontheimer EJ: Origins and Mechanisms of miRNAs and siRNAs. Cell 136: 642-655, 2009.

14. Schmittgen TD: Regulation of microRNA processing in development, differentiation and cancer. J Cell Mol Med 12: 1811-1819, 2008.

15. Rosenfeld N, Aharonov R, Meiri E, Rosenwald S, Spector Y, Zepeniuk M, Benjamin H, Shabes N, Tabak S, Levy A, et al: MicroRNAs accurately identify cancer tissue origin. Nat Biotechnol 26: 462-469, 2008.

16. Liang Y, Ridzon D, Wong L and Chen C: Characterization of microRNA expression profiles in normal human tissues. BMC Genomics 8: 166, 2007.

17. Croce CM: Causes and consequences of microRNA dysregulation in cancer. Nat Rev Genet 10: 704-714, 2009.

18. Mathé EA, Nguyen GH, Bowman ED, Zhao Y, Budhu A, Schetter AJ, Braun R, Reimers M, Kumamoto K, Hughes D, et al: MicroRNA expression in squamous cell carcinoma and adenocarcinoma of the esophagus: Associations with survival. Clin Cancer Res 15: 6192-6200, 2009.

19. Feber A, Xi L, Luketich JD, Pennathur A, Landreneau RJ, Wu M, Swanson SJ, Godfrey TE and Litle VR: MicroRNA expression profiles of esophageal cancer. J Thorac Cardiovasc Surg 135: 255-260, 2008

20. Lebanony D, Benjamin H, Gilad S, Ezagouri M, Dov A, Ashkenazi K, Gefen N, Izraeli S, Rechavi G, Pass H, et al: Diagnostic assay based on I-miR-205 expression distinguishes squamous from nonsquamous non-small-cell lung carcinoma. J Clin Oncol 27: 2030-2037, 2009. 
21. Kimura S, Naganuma S, Susuki D, Hirono Y, Yamaguchi A, Fujieda S, Sano K and Itoh H: Expression of microRNAs in squamous cell carcinoma of human head and neck and the esophagus: miR-205 and miR-21 are specific markers for HNSCC and ESCC. Oncol Rep 23: 1625-1633, 2010.

22. Smith CM, Michael MZ, Watson DI, Tan G, Astill DS Hummel R and Hussey DJ: Impact of gastro-oesophageal reflux on microRNA expression, location and function. BMC Gastroenterol 13: 4, 2013.

23. Leeuwenburgh I, Haringsma J, Van Dekken H, Scholten P, Siersema PD and Kuipers EJ: Long-term risk of oesophagitis, Barrett's oesophagus and oesophageal cancer in achalasia patients. Scand J Gastroenterol Suppl 7-10, 2006.

24. Descriptive Rules for Achalasia of the Esophagus, 4th Edition. Japan Society of Esophageal Diseases, Kanehara \& Co., Ltd., Tokyo, 2012.

25. Matsui N, Akahoshi K, Nakamura K, Ihara E and Kita H: Endoscopic submucosal dissection for removal of superficial gastrointestinal neoplasms: A technical review. World J Gastrointest Endosc 4: 123-136, 2012.

26. Gibcus JH, Menkema L, Mastik MF, Hermsen MA, de Bock GH, van Velthuysen ML, Takes RP, Kok K, Alvarez Marcos CA, van der Laan BF, et al: Amplicon mapping and expression profiling identify the Fas-associated death domain gene as a new driver in the 11q13.3 amplicon in laryngeal/pharyngeal cancer. Clin Cancer Res 13: 6257-6266, 2007.

27. Reshmi SC, Huang X, Schoppy DW, Black RC, Saunders WS Smith DI and Gollin SM: Relationship between FRA11F and 11q13 gene amplification in oral cancer. Genes Chromosomes Cancer 46: 143-54, 2007.

28. Cheng CK, Chow LW, Loo WT, Chan TK and Chan V: The cell cycle checkpoint gene Rad9 is a novel oncogene activated by 11q13 amplification and DNA methylation in breast cancer. Cancer Res 65: 8646-8654, 2005.

29. Zheng SL, Stevens VL, Wiklund F, Isaacs SD, Sun J, Smith S, Pruett K, Wiley KE, Kim ST, Zhu Y, et al: Two independent prostate cancer risk-associated Loci at 11q13. Cancer Epidemiol Biomarkers Prev 18: 1815-1820, 2009.
30. Chen Y and Gorski DH: Regulation of angiogenesis through a microRNA (miR-130a) that down-regulates antiangiogenic homeobox genes GAX and HOXA5. Blood 111: 1217-1226, 2008.

31. Boll K, Reiche K, Kasack K, Mörbt N, Kretzschmar AK, Tomm JM, Verhaegh G, Schalken J, von Bergen M, Horn F and Hackermüller J: MiR-130a, miR-203 and miR-205 jointly repress key oncogenic pathways and are downregulated in prostate carcinoma. Oncogene 32: 277-285, 2013.

32. Xu N, Shen C, Luo Y, Xia L, Xue F, Xia Q and Zhang J: Upregulated miR-130a increases drug resistance by regulating RUNX3 and Wnt signaling in cisplatin-treated HCC cell. Biochem Biophys Res Commun 425: 468-272, 2012.

33. Yang L, Li N, Wang H, Jia X, Wang X and Luo J: Altered microRNA expression in cisplatin-resistant ovarian cancer cells and upregulation of miR-130a associated with MDR1/P-glycoprotein-mediated drug resistance. Oncol Rep 28: 592-600, 2012.

34. Acunzo M, Visone R, Romano G, Veronese A, Lovat F, Palmieri D, Bottoni A, Garofalo M, Gasparini P,Condorelli G, et al: miR-130a targets MET and induces TRAIL-sensitivity in NSCLC by downregulating miR-221 and 222. Oncogene 31: 634-642, 2012.

35. Shi B, Gao H, Zhang T and Cui Q: Analysis of plasma microRNA expression profiles revealed different cancer susceptibility in healthy young adult smokers and middle-aged smokers. Oncotarget 7: 21676-21685, 2016

36. Mullany LE, Herrick JS, Wolff RK, Stevens JR and Slattery ML: Assosiation of cigarette smoking and microRNA expression in rectal cancer: Insight into tumor phenotype. Cancer Epidemiol 45: 98-107, 2016.

37. Laporte J, Hu LJ, Kretz C, Mandel JL, Kioschis P, Coy JF, Kluack SM, Poustka A and Dahl N: A gene mutated in X-linked myotubular myopathy defines a new putative tyrosine phosphatase family conserved in yeast. Nat Genet 13: 175-182, 1996.

38. Senderek J, Bergmann C, Weber S, Ketelsen UP, Schorle H, Rudnik-Shöneborn S, Büttner R, Buchheim E and Zerres K: Mutation of the SBF2 gene, encoding a novel member of the myotubularin family, in Charcot-Marie-Tooth neuropathy type 4B2/11p15. Hum Mol Genet 12: 349-356, 2003. 